\title{
Prolonged Mechanical Ventilation Predictors in Patients Undergoing Liver Transplantation
}

(i) Meltem Güner CAN11, iD Ali ÖZER²

${ }^{1}$ Acıbadem Mehmet Ali Aydınlar University Atakent Hospital, Department of Anesthesia and Reanimation, İstanbul, Turkey

${ }^{2}$ Acıbadem Mehmet Ali Aydınlar University Atakent Hospital, Department of Organ Transplantation, İstanbul, Turkey

\section{ABSTRACT}

Objective: Liver transplantation is the current treatment modality for end-stage liver disease. While there is increasing drive towards early extubation in patients undergoing liver transplantation, prolonged meachanical ventilation (PMV) related to various factors can be observed. We tried to determine the risk factors associated with PMV.

Methods: One hundred twenty five patients (age>18 years) of with the American Society of Anesthesiology (ASA) III-IV group undergoing liver transplantation were enrolled in this retrospective study. Patient charts, intraoperative and intensive care unit follow-up forms, and electronic medical recording system were used for data collection. Patients were categorized as having received (with) or not received (without) prolonged mechanical ventilation ( $>24$ hours), and perioperative risk factors were attempted to determine.

Results: No significant intergroup differences were found in demographic variables but ASA and model for end-stage liver disease (MELD) scores were significantly higher in the with- than in the without-PMV group. Total amount of suspension of erythrocytess suspension, fresh frosen plasm, and cell-saver blood used intraoperatively was higher in the with-PMV group but difference did not reach statistical significance. Patients with-PMV had significantly higher cryoprecipitate transfusion rates than did those without-PMV ( $\mathrm{p}=0.007$ ). While no significante intergroup differences were found in mortality and lenght of hospital stay; lenght of intensive care unit (ICU) stay was higher in the with- PMV group ( $\mathrm{p}=0.001$ ). Extravascular lung water index, global ecosystem dynamics investigation, and pulse volume index values obtained from pulse contour cardiac output (PiCCO) monitorization were significantly lowver in the with- than in the without-PMV group.

Conclusion: We found that preoperative high ASA and MELD scores, high blood product transfusion rates, and hypovolemia supported by the PiCCO measurements are were closely related to PMV and long lenght of ICU stay.

Keywords: Liver transplatation, weaning, prolonged mechanical ventilation

\section{Introduction}

Liver transplantation is a safe and effective way of treating end-stage liver disease (ESLD) due to better understanding of the pathology of liver diseases, the development of organ protection techniques, the use of safer and effective immunosuppressive drugs, advances in surgical techniques, anesthesia and monitorization. With these improvements, both perioperative complication and mortality rates have decreased significantly, and 1-year life expectancy has exceeded $90 \%$.
Liver transplant candidates are often characterized by a hyperdynamic circulatory state of cirrhotic cardiomyopathy (1) and a complex process in which all systems are affected. Need for massive transfusion due to severe surgical blood loss and intravascular volume shift, surgical large vein maneuvers and the effects of current coagulopathy, metabolic dysfunction, hemodynamic instability and reperfusion on systems require intraoperative detailed monitorization (2). In addition to standard monitorization, cardiac output monitorization is required for the

Address for Correspondence: Ali ÖZER, Acıbadem Mehmet Ali Aydınlar University Atakent Hospital, Department of Organ Transplantation, İstanbul, Turkey

Phone: +90 5332122324 E-mail: draliozerr@gmail.com ORCID ID: orcid.org/0000-0003-4736-3418 
management of intraoperative hemodynamic changes. For this reason, nowadays, pulse contour cardiac output (PiCCO) ve hemodynamic monitor methods which combine thermodilution and arterial waveform analysis methods, have less complication rates than pulmonary artery catheter and are less invasive, have gained importance. PiCCO, also used in our clinic on a routine basis, is an invasive hemodynamic monitoring method which uses the combination of these two techniques and shows important parameters such as cardiac index (CI), stroke volume index (SVI), global end-diastolic index (GEDI), stroke volume variation (SVV), global ejection fraction, systemic vascular resistance index (SVRI), extravascular lung water index (ELWI) and pulmonary vascular permeability index (PVPI).

Although early extubation trend increases in most of the centers where liver transplantation is performed due to improvements in perioperative care $(3,4)$, prolonged mechanical ventilation is required due to various factors such as massive blood transfusion, pulmonary edema, lung damage, acid, capillary leakage, primary pulmonary and heart disease (5). In this study, we aimed to determine the risk factors associated with prolonged mechanical ventilation requirement in patients undergoing liver transplantation.

\section{Methods}

\section{Anesthesia}

After obtaining ethics committee approval, American Society of Anesthesiologists (ASA) III-IV 125 patients aged over 18 years who underwent liver transplantation between 2015 and 2017 were included in this retrospective study. Patients whose preoperative anesthetic evaluations were completed one day before, were taken to the operation room without premedication and heart rate, noninvasive blood pressure, $\mathrm{SpO}_{2}$, body temperature and $\mathrm{EtCO}_{2}$ monitorizations were performed. After induction of anesthesia, invasive arterial pressure, central venous pressure (with right internal jugular catheterization accompanied by ultrasound) and PiCCO monitorization (with right femoral artery catheterization using the existing central venous catheter and PiCCO catheter with special thermistor) were performed. Propofol and fentanyl was used for induction of anesthesia and cis-atracurium was used for muscle relaxation. After endotracheal intubation, desfluran (6-8\%) was given in 40-50\% oxygen-air mixture and intravenous infusion of remifentanyl [0.05-0,25 $\mathrm{g} \mathrm{kg} \mathrm{k}^{-1}$ minimum $\left.(\mathrm{min})^{-1}\right]$ was used for maintenance of anesthesia. Muscle relaxation was maintained with cis-atracurium infusion. Mechanical ventilation parameters were adjusted to keep $\mathrm{PaCO}_{2}$ values between 30-35 $\mathrm{mmHg}$, tidal volume $6-8 \mathrm{~mL} \mathrm{~kg}^{-1}$, respiration rate $10-14 / \mathrm{min}$ and peep 4-6 mmHg. Restrictive fluid management was performed in patients. The replacement of albumin was determined according to the level of albumin of the patients. Erythrocyte suspension (ES) replacement was performed to keep hematocrit values at $26-28 \%$. In our study, thromboelastography was not used and fresh frozen plasma (FFP), cryopresipitat or platelet replacement (complete blood count and prothrombin time, activated partial thromboplastin time, international normalized ratio and fibrinogen levels measured in standard dissection phase and anhepatic and neohepatic phases). Inotrop/vasopressor agents were initiated due to the data of invasive hemodynamic monitorization (blood pressure, heart rate, cardiac flow, SVRI) and the presence of hemodynamic instability. The patients were heated with lower heating blankets and blown heating systems. In addition, hypotermia was prevented by using fluid heating systems for all intravenous fluids and blood products. In order to prevent rejection, all patients were administered 10 $\mathrm{mg} \mathrm{kg}{ }^{-1}$ methyl prednisolone intravenously prior to perfusion during anhepatic period. Blood glucose levels of patients were kept between 80-160 mg/dL. Acıbadem Mehmet Ali Aydınlar University ATADEK-2017/16.

\section{Surgical Technique}

The patients were operated with bilateral subcostal incision or inverted L incision. Greft implantation was started with the main hepatic vein (left/right hepatic vein) and vena cava anastomoses were performed under partial clamp using piggy-back method. Then, if available, other vein anastomoses (segment 5-8 or inferior vein) were made by parachute technique on openings on vena cava. Portal vein anastomosis was performed end-toend, if possible and anhepatic phase was completed and graft perfusion stage was initiated. Following perfusion, the hepatic artery anastomosis was made preferably anatomical and in case of a problem in recipient artery, extra-anatomic arterial anastomosis was made. Gallbladder anastomosis was performed by end-to-end/hepatico-jejunostomy method. In none of the patients portacaval shunt was used. After anastomoses were ultrasonographically shown as open and flow was shown as good by the radiologist, bleeding control was done and surgery was completed.

\section{Intensive Care and Extubation}

The patients were sent to intensive care unit as intubated at the end of the operation and they were followed up with electrocardiography, heart rate, invasive blood pressure, central venous pressure, $\mathrm{EtCO}_{2}$ and temperature monitorizations. Analgesia and sedation management were performed for each patient in accordance with the patient's own intensive care process. When intensive care patients met the standard criteria (Table 1), they were extubated with T-tube method, one of spontaneous breathing methods (Table 2) by the intensive care team (6). Mechanical ventilation was accepted as prolonged mechanical ventilation when the duration of the ventilation exceeded 24 hours.

\section{Data Collection}

Anesthesia preoperative evaluation forms, intraoperative monitoring forms, intensive care monitoring forms, consultation notes, and epicrisis were examined and the following data were recorded.

Preoperative Data: Demographic data, comorbid diseases, smoking habit, etiology of end-stage liver disease, model for end-stage liver disease (MELD) scores, presence and degree of preoperative encephalopathy, presence of hepatopulmonary and hepatorenal syndrome. 
Intraoperative Data: Intraoperative fluid amounts, blood and blood product transfusion amounts, hemodynamic data (heart rate, invasive arterial pressures, central venous pressures) and PiCCO data (CI, SVI, SVV, GEDI, SVRI ELWI, PVPI), inotropvasopressor needs, anhepatic phase and surgery durations.

Postoperative Data: Duration on mechanical ventilator in intensive care unit and stay in intensive care unit-hospital, need for retransplantation, mortality and morbidity.

Patients were divided into two groups based on the time periods for mechanical ventilation determined in the previous studies $(7,8)$ : Patients needing prolonged mechanical ventilation ( $>24$ hours) and patients not needing prolonged mechanical ventilation $(<24$ hours) and the risk factors for prolonged duration of mechanical ventilation were determined.

\section{Table 1. Standard extubation criteria}

- Adequate oxygenation $\left(\mathrm{PO}_{2}>60 \mathrm{mmHg}\right.$ when $\mathrm{FiO}_{2}<0.4$ and PEEP: $5-10 \mathrm{~cm} \mathrm{H}_{2} \mathrm{O}$, $\mathrm{PO}_{2} / \mathrm{FiO}_{2}>150-300$

- Stable hemodynamic status (heart rate $<140$ beats/min, stable blood pressure without vasopressor or with minimal vasopressor support)

Objective criteria

Subjective clinical evaluation

- Fever $<38^{\circ} \mathrm{C}$

- The absence of respiratory acidosis

- Adequate hemoglobin level (8-10 g/dL)

- Stable mental status (GCS $>13$, no sedative infusion)

- Stable metabolic status (electrolyte, blood glucose...)

- Amelioration of acute phase of disease

- Clinician considering suitability for extubation

- Adequate cough reflex

GCS: Glasgow coma scale, PEEP: Positive end-expiratory pressure

\section{Statistical Analysis}

Statistical analysis was performed using the SPSS Statistics for Windows, version 21 (IBM Corp., Armonk, NY). Two-tailed tests were used in all statistical analyses. In comparisons between the two groups, independent samples $t$ test was used in evaluation of parametric data and Pearson's chi-square and Fisher's exact test, if needed, were used in evaluation of non-parametric data. The relationship between etiological factors and prolonged mechanical ventilation was examined by Bivariate correlation test. Categorical data were expressed as mean \pm standard deviation, non-categorical data were expressed as number (n) and percent (\%). A p value less than 0.05 was considered statistically significant.

\section{Results}

The data and files of 125 patients over 18 years of age who underwent liver transplantation from living donor were retrospectively analyzed. All data of 10 patients could be reached and 115 patients were included in the study. In 10 (8.69\%) of 115 patients, prolonged mechanical ventilation need was observed. Demographic data and ASA and MELD scores of the patients were summarized in Table 3. ASA and MELD scores were significantly higher in the prolonged mechanical ventilation group than in the other group. There was no difference between the groups in terms of the presence of comorbid disease, hepatopulmonary syndrome, hepatorenal syndrome, portal hypertension and hepatic encephalopathy and etiological factors.

When intraoperative variables were examined, there was no significant difference between the groups in terms of the amount of crystalloid and albumin given with restrictive liquid approach. Although ES, FFP, cell saver blood and platelet amounts were higher in the prolonged mechanical ventilation group, statistical significance was not found (Figure 1). The amount of cryoprecipitate used $(1.67 \pm 3.39 \mathrm{U})$ was significantly higher in the prolonged mechanical ventilation group than in the other

Table 2. Weaning methods

Method

T-tube method

Pressure support ventilation

(PSV)

Synchronized intermittent mandatory ventilation

(SIMV)

CPAP

\section{Advantage}

- Testing patient's spontaneous breathing ability

- Allow working/resting periods

- Faster weaning than SIMV

- Synchronization

- Increased patient comfort

- Slow loading of respiratory work on the patient

- Faster weaning than SIMV

- Prevention of muscle fatigue

- Guaranteed minimum minute ventilation

- Use of alarm systems

- Applicability with PSV/CPAP

- Allowing tidal volume monitoring
Disadvantage

- Sudden loading of respiratory work to patient

- Endotracheal tube resistance

- Disabled alarm systems

- Careful observation requirement

- Observation of major changes in minute ventilation
- Desynchronization
- Long weaning process
- Increased muscle fatigue
- Sudden loading of respiratory work to patient

SIMV: Synchronized intermittent mandatory ventilation, PSV: Pressure support ventilation, CPAP: Continious positive airway pressure 
group $(0.21 \pm 1.27 \mathrm{U})(\mathrm{p}=0.007)$. The duration of the surgery was $495 \pm 76 \mathrm{~min}$, the duration of the anhepatic phase was $95 \pm 41 \mathrm{~min}$ in the prolonged mechanical ventilation group and the duration of the surgery was $529 \pm 125 \mathrm{~min}$, the duration of the anhepatic phase was $90 \pm 34 \mathrm{~min}$ in the other group. Differences in terms of duration of the surgery and the anhepatic phase between groups were not statistically significant.

The results of PiCCO measurements in dissection phase, anhepatic phase and neohepatic phase were summarized in Table 4.

In our study, 10 of 115 patients died and one had a need for retransplantation. There was no difference between the groups in terms of mortality and hospital stay, whereas the duration of stay in intensive care unit was $8.10 \pm 9.0$ days in the prolonged mechanical ventilation group and $3.13 \pm 3.51$ days in the other group $(\mathrm{p}=0.001)$.

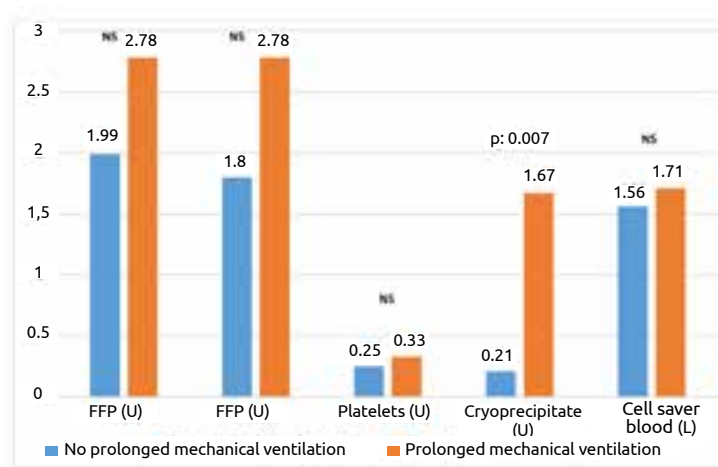

Figure 1. Blood product utilization rates in patients undergoing liver transplantation

ES: Erythrocyte suspension, FFP: Fresh frozen plasma, NS: Not significant

Table 3. Demographic data and risk scores

\begin{tabular}{|l|l|l|l|}
\hline & $\begin{array}{l}\text { Prolonged mechanical ventilation } \\
\text { No }(n=105)\end{array}$ & Yes $(n=10)$ & 0.558 \\
\hline Age (year) & $49.61 \pm 12.64$ & $47.10 \pm 16.03$ & 0.164 \\
\hline Weight $(\mathbf{k g})$ & $73.99 \pm 15.75$ & $66.35 \pm 23.20$ & 0.507 \\
\hline Height $(\mathbf{c m})$ & $168.07 \pm 12.48$ & $165.30 \pm 13.87$ & 0.179 \\
\hline BMI (kg/m) & $25.75 \pm 4.77$ & $23.56 \pm 6.36$ & 0.002 \\
\hline ASA $(\mathbf{I}-\mathbf{V})$ & $3.18 \pm 0.386$ & $3.60 \pm 0.516$ & 0.013 \\
\hline MELD & $18.71 \pm 4.24$ & $22.55 \pm 5.89$ & \\
\hline ASA: American Society of Anesthesiologists, MELD: Model for end-stage liver disease, BMI: Body mass index & \\
\hline
\end{tabular}

Table 4. Intraoperative hemodynamic monitorization data in patients undergoing liver transplantation

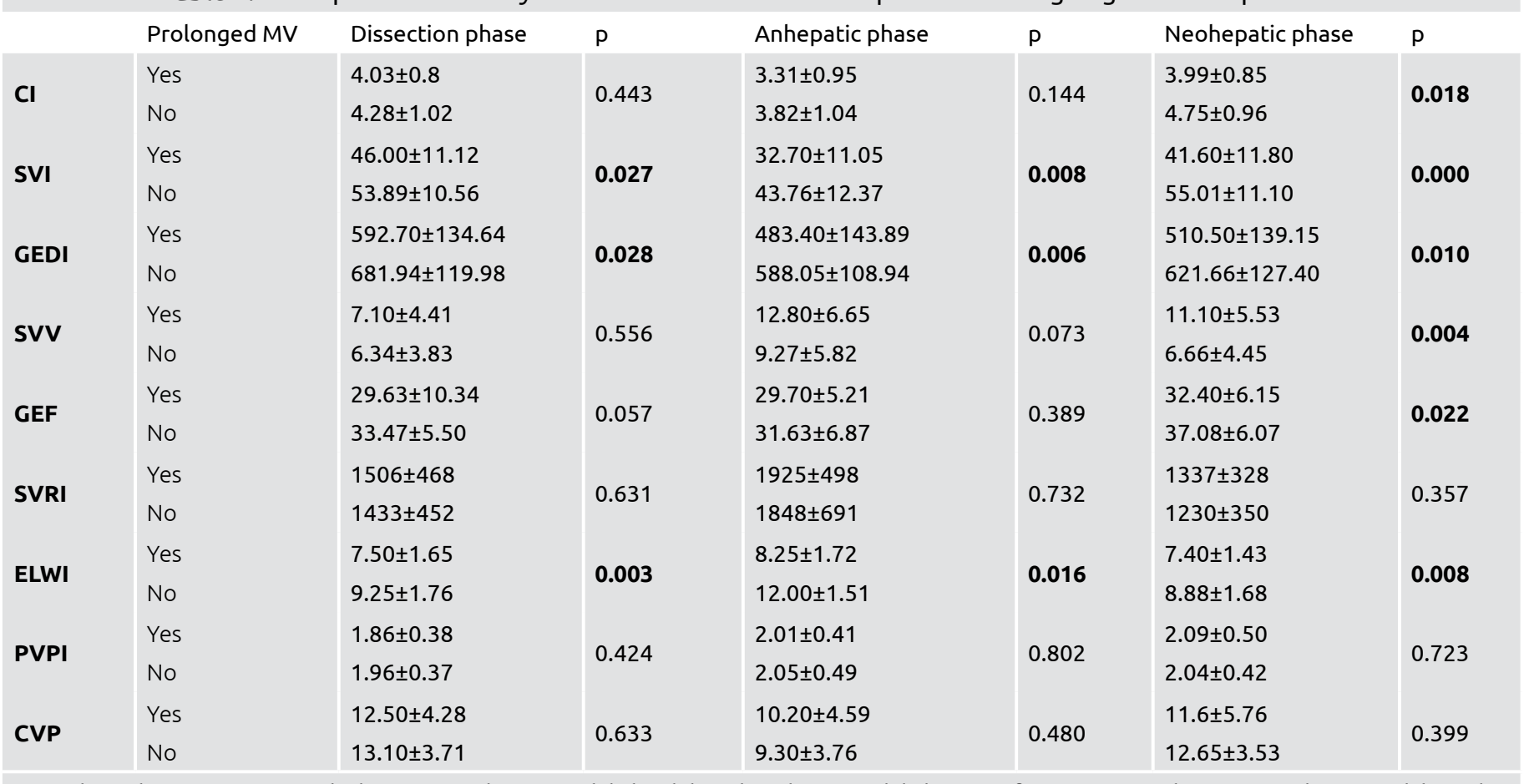

CI: Cardiac index, ELWI: Extravascular lung water index, GEDI: Global end-diastolic index, GEF: Global ejection fraction, PVPI: Pulmonary vascular permeability index, SVI: Stroke volume index, SVRI: Systemic vascular resistance index, SVV: Stroke volume variation, CVP: Entral venous pressure MV: Mechanical ventilation 


\section{Discussion}

In patients undergoing major surgery, it may be necessary to maintain mechanical ventilation support in intensive care unit. The duration of prolonged mechanical ventilation in these patients is accepted as 48 hours in some clinical studies $(9,10)$ and as 24 hours in some studies $(7,8)$. In our study, we accepted mechanical ventilation for more than 24 hours as "prolonged mechanical ventilation need". Prolonged mechanical ventilation after major surgery is found to be associated with increased morbidity and mortality $(11,12)$. The need for prolonged mechanical ventilation in patients undergoing liver transplantation, although traditionally considered as a postoperative pulmonary complication, may occur as a result of graft dysfunction, impairment of consciousness, preoperative sedentary condition, hemodynamic instability, bleeding diathesis, residual anesthetic effect, heart failure and prolonged surgical process. For this reason, general practice in patients undergoing liver transplantation during the postoperative period is to monitor the patient in the mechanical ventilator for a while.

Weaning is the gradual reduction of mechanical ventilator support until the patient has spontaneous and sufficient breathing. In this process, the physician should gradually increase the patient's effort, reduce mechanical ventilator support, and initiate the weaning process at the appropriate time. Weaning types are summarized in Table 2 . Studies show that early and inappropriate weaning tryings are associated with increased cardiac ischemia risk and prolonged mechanical ventilation durations. When appropriate clinical and laboratory findings were obtained and when the weaning indexes were suitable for extubation in our intensive care unit, patients were extubated frequently using T-tube method.

In our study, we found that postoperative mechanical ventilation support was prolonged ( $>24$ hours) in $8.69 \%$ of adult patients undergoing liver transplantation. The preoperative ASA and MELD scores and ES, FFP, cell saver blood, platelet and cryoprespit amounts used in the operation were higher in the prolonged mechanical ventilation group than the other group.

In patients undergoing liver transplantation, it may be necessary to give blood and blood products in serious amounts to the patients for various reasons during the intraoperative and postoperative periods. Therefore, high bleeding rate and high amount of transfusion of blood products during perioperative period are among the most important factors responsible for prolongation of mechanical ventilation support and increased mortality and morbidity after liver transplantation (13). In a recent study by Prasad et al. (14) the volume of blood products used in patients undergoing orthotopic liver transplantation was found to be a significant predictor of postoperative poor outcome. In our study, although the amount of transfusion of erythrocyte suspension and FFP was very low compared with the other studies $(15,16)$, it was found to be higher in the prolonged mechanical ventilation group than the other group without reaching statistical significance. On the other hand, the use of cryopresipitat was found to be statistically significantly higher in the prolonged mechanical ventilation group. Therefore, we believe that high blood transfusion rates should be a stimulant for prolonged mechanical ventilation, so that blood conservation techniques and intraoperative autologous transfusions will affect patient outcomes.

Pulmonary edema, another risk factor for prolonged mechanical ventilation after liver transplantation, may be hydrostatic or nonhydrostatic. Alveolocapillary membrane integrity is impaired and this impairment may be aggravated by ischemia-reperfusion damage during transplantation in patients with $\operatorname{ESLD}(17,18)$. The clinical outcome is the increase of protein-rich extravascular fluid in the lung. Measurement of parameters showing the integrity of this liquid and alveolocapillary membrane may provide important information to clinician. In the literature, there are studies showing that ELWI and PVPI values obtained by PiCCO monitoring had prognostic value in terms of results in critical patients, and that PVPI values could be used effectively to differentiate hydrostatic pulmonary edema from non-hydrostatic pulmonary edema (19-21). In those study, high ELWI levels were associated with decreased survival, and PVPI was defined as a good indicator for results in patients in intensive care units. However, studies on the use of these parameters during the perioperative period are limited. Garutti and colleagues showed that ELWI values $\geq 12 \mathrm{~mL} / \mathrm{kg}$ and PVPI values $\geq 2.3$ obtained by PiCCO measurements made at the end of surgery in patients with liver transplantation, were associated with longer mechanical ventilation and longer duration of hospital stay (22). In our study, we found that ELWI values were lower in all three phases of transplantation in the prolonged mechanical ventilation group than the other group and that there was no significant difference between the two groups in terms of PVPI values. In support of these results, GEDI ve SVI values obtained in all three phases of surgery were found to be lower in the prolonged mechanical ventilation group. Contrary to the results of the studies in the literature which showed that high ELWI, PVPI and GEDI levels were risk factors for prolonged mechanical ventilation; low values found in our study seems to be a stimulating factor. Prolonged mechanical ventilation may be due to having lower intravascular volumes of these patients or it may be due to having greater fluid and blood transfusion need during surgery in these patients, as well as it may be due to organ damage caused by hypoperfusion due to inadequate fluid resuscitation.

Another risk factor for prolonged mechanical ventilation is preoperative poor condition. There are many studies in the literature that show that high ASA and MELD scores are associated with poor outcomes $(14,15,23)$. In our study, ASA and MELD scores were significantly higher in the prolonged mechanical ventilation group, but the effect of age, gender and comorbid disease on mechanical ventilation duration was not shown.

There were a number of limitations in our study. One of these was the retrospective design of our study. In addition to this, a low number of patients who needed prolonged mechanical ventilation might be a parameter that seemed to affect the statistical value of the results. In addition, the results of arterial wave analysis and 
PiCCO measurements might have been unexpectedly affected by vascular impedance and compliance, which have been impaired in ESLD. It is well known that the current-dependent invasive hemodynamic monitorization methods have no proven effects on the results, mortality and morbidity.

\section{Study Limitations}

One limitation of our study was the retrospective design of the study. In addition, the dose and duration of the analgesic and sedative agents, which directly affect the duration of ventilation support, were not known. However, patients undergoing liver transplantation in our center were monitored by the same team using standard protocols in intensive care unit.

\section{Conclusion}

We observed that preoperatively high ASA and MELD scores in patients undergoing liver transplantation, and the use of blood and blood products in intraoperative period and hypovolemia observed in PiCCO measurements extended the duration of stay in mechanical ventilation and in intensive care unit during the postoperative period. Since prolonged duration of mechanical ventilation and stay in the intensive care unit are known to be directly correlated with morbidity and mortality; the determination of factors that constitute a risk to the need for prolonged mechanical ventilation and the determination of appropriate treatment strategies by taking the necessary measures will reduce the duration of hospitalization in the intensive care unit and mortality and morbidity rates. We believe that there is a need to work more for it.

\section{Ethics}

Ethics Committee Approval: Acıbadem Mehmet Ali Aydınlar University ATADEK-2017/16.

Informed Consent: Retrospective study.

Peer-review: Externally peer-reviewed.

\section{Authorship Contributions}

Concept: M.G.C., Design: M.G.C., A.Ö., Data Collection or Processing: M.G.C., A.Ö., Analysis or Interpretation: M.G.C., A.Ö., Literature Search: M.G.C., Writing: M.G.C., A.Ö.

Conflict of Interest: No conflict of interest was declared by the authors.

Financial Disclosure: The authors declared that this study received no financial support.

\section{References}

1. Siniscalchi A, Aurini L, Spedicato S, Bernardi E, Zanoni A, Dante A, et al. Hyperdynamic circulation in cirrhosis: predictive factors and outcome following liver transplantation. Minerva Anestesiol 2013;79:15-23.

2. De Wolf AM. Pulmonary artery catheter: rest in peace? Not just quite yet. Liver Transpl 2008;14:917-8.
3. Zeyneloglu P, Pirat A, Guner M, Torgay A, Karakayali H, Arslan G. Predictors of immediate tracheal extubation in the operating room after liver transplantation. Transplant Proc 2007;39:1187-9.

4. Mandell MS, Stoner TJ, Barnett R, Shaked A, Bellamy M, Biancofiore $\mathrm{G}$, et al. A multicenter evaluation of safety of early extubation in liver transplant recipients. Liver Transpl 2007;13:1557-63.

5. Lee JM, Chang HW, Park CS, Park HJ, Kim JE, Choi JH. Evaluation of mechanical ventilation and its influencing factors between the living related liver transplantation and cadaveric whole liver transplantation. Korean J Anesthesiol 2005;49:816-21.

6. MacIntyre NR, Cook DJ, Ely EW Jr, Epstein SK, Fink JB, Heffner

$\mathrm{JE}$, et al. Evidence-based guidelines for weaning and discontinuing ventilatory support: a collective task force facilitated by the American College of Chest Physicians; the American Association for Respiratory Care; and the American College of Critical Care Medicine. Chest 2001;120:375-95.

7. Glanemann M, Langrehr J, Kaisers U, Schenk R, Müller A, Stange $\mathrm{B}$, et al. Postoperative tracheal extubation after orthotopic liver transplantation. Acta Anaesthesiol Scand 2001;45:333-9.

8. Choi JH, Kim TH, Lee JM. Evaluation of usefulness of perioperative risk factors which affect early or delayed extubation after liver transplantation. Korean J Anesthesiol 2003;44:847-52.

9. Mandell MS, Campsen J, Zimmerman M, Biancofiore G, Tsou MY. The clinical value of early extubation. Curr Opin Organ Transplant 2009;14:297-302.

10. Neelakanta G, Sopher M, Chan S, Pregler J, Steadman R, Braunfeld $\mathrm{M}$, et al. Early tracheal extubation after liver transplantation. J Cardiothorac Vasc Anesth 1997;11:165-7.

11. O'Meara ME, Whiteley SM, Sellors JM, Luntley JM, Davison $\mathrm{S}$, McClean $\mathrm{P}$, et al. Immediate extubation of children following liver transplantation is safe and may be beneficial. Transplantation 2005;80:959-63.

12. Rajakaruna C, Rogers CA, Angelini GD, Ascione R. Risk factors for and economic implications of prolonged ventilation after cardiac surgery. J Thorac Cardiovasc Surg 2005;130:1270-7.

13. Schrem H, Klußmann A, Focken M, Emmanouilidis N, Oldhafer F, Klempnauer J, et al. Post-operative hemorrhage after liver transplantation: risk factors and long-term outcome. Ann Transplant 2016;21:46-55.

14. Prasad V, Guerrisi M, Dauri M, Coniglione F, Tisone G, De Carolis $\mathrm{E}$, et al. Prediction of postoperative outcomes using intraoperative hemodynamic monitoring data. Sci Rep 2017;7:16376.

15. Lee S, Jung HS, Choi JH, Lee J, Hong SH, Lee SH, et al. Perioperative risk factors for prolonged mechanical ventilation after liver transplantation due to acute liver failure. Korean J Anesthesiol 2013;65:228-36.

16. Yıldız I, Sabuncuoğlu MZ, Koca YS, Solmaz Alkaya F, Şenol A. Yeni kurulan organ nakli merkezimizde yapılan karaciğer nakli sonuçlarımız. Sdü Sağlık Bilimleri Enstitüsü Dergisi 2017;8:1820. 
17. Schraufnagel DE, Malik R, Goel V, Ohara N, Chang SW. Lung capillary changes in hepatic cirrhosis in rats. Am J Physiol 1997;272:139-47.

18. Chang SW, Ohara N. Increased pulmonary vascular permeability in rats with biliary cirrhosis: role of thromboxane A2. Am J Physiol 1993;264:245-52.

19. Brown LM, Liu KD, Matthay MA. Measurement of extravascular lung water using the single indicator method in patients: research and potential clinical value. Am J Physiol Lung Cell Mol Physiol 2009;297:547-58.

20. Kushimoto S, Taira Y, Kitazawa Y, Okuchi K, Sakamoto T, Ishikura $\mathrm{H}$, et al. The clinical usefulness of extravascular lung water and pulmonary vascular permeability index to diagnose and characterize pulmonary edema: a prospective multicenter study on the quantitative differential diagnostic definition for acute lung injury/ acute respiratory distress syndrome. Crit Care 2012;16:232.

21. Jozwiak M, Silva S, Persichini R, Anguel N, Osman D, Richard C, et al. Extravascular lung water is an independent prognostic factor in patients with acute respiratory distress syndrome. Crit Care Med 2013;41:472-80.

22. Garutti I, Sanz J, Olmedilla L, Tranche I, Vilchez A, FernandezQuero L, et al. Extravascular lung water and pulmonary vascular permeability index measured at the end of surgery are independent predictors of prolonged mechanical ventilation in patients undergoing liver transplantation. Anesth Analg 2015;121:736-45.

23. Nafiu OO, Carello K, Lal A, Magee J, Picton P. Factors associated with postoperative prolonged mechanical ventilation in pediatric liver transplant recipients. Anesthesiol Res Pract 2017;2017:3728289. 